\title{
Corneal Collagen Cross-Linking Combined with an Artiflex Iris-Fixated Anterior Chamber Phakic Intraocular Lens Implantation in a Patient with Progressive Keratoconus
}

\author{
Sharif Hashmani ${ }^{\mathrm{a}} \quad$ Nauman Hashmani $^{\mathrm{a}} \quad$ Roha Saeed Memon ${ }^{\mathrm{b}}$ \\ ${ }^{a}$ Hashmanis Hospital, Karachi, Pakistan; ${ }^{b}$ Dow Medical College, Karachi, Pakistan
}

\section{Keywords}

Refractive surgery · Keratoconus · Corneal cross-linking · Phakic intraocular lens

\begin{abstract}
We present here the case of a 24-year-old male who experienced progressive keratoconus and vision loss which adversely affected his ability to carry out everyday tasks. This landed him in the Hashmanis Hospital for consultation. He had a preoperative best corrected visual acuity of $6 / 12$. He underwent multiple Oculus Pentacam examinations, which showed progressive keratoconus. Corneal collagen cross-linking $(C X L)$ was performed to stabilize his cornea and, subsequently, an Artiflex anterior chamber iris-fixated phakic intraocular lens (ACIF-PIOL) was implanted to alleviate his refractive errors. The patient achieved a postoperative uncorrected visual acuity of $6 / 12$. This report shows that CXL combined with ACIFPIOL can be safe and effective in those with progressive keratoconus.
\end{abstract}




\section{Case Reports in Ophthalmology}

\begin{tabular}{l|l}
\hline Case Rep Ophthalmol 2017;8:482-488 \\
\hline DOI: $10.1159 / 000480728$ & $\begin{array}{l}\text { (c) 2017 The Author(s). Published by S. Karger AG, Basel } \\
\text { www.karger.com/cop }\end{array}$ \\
\hline
\end{tabular}

Hashmani et al.:Phakic Intraocular Lens in Progressive Keratoconus

\section{Introduction}

The cornea is a clear window which refracts light entering the eye. Abnormal structural changes within the normally dome-shaped cornea such as thinning and conical bulging can lead to an eye disease called keratoconus (KC) [1]. KC is a progressive, degenerative and noninflammatory disease that causes abnormal structural changes to the cornea, which can cause myopia, astigmatism [2], and corneal scarring. These atypical changes can lead to visual impairment depriving individuals from carrying out normal day-to-day tasks.

$\mathrm{KC}$ is a multifactorial disease where exposure to ultraviolet (UV) rays from the sun and chronic eye irritation are considered potential causes. Susceptible individuals are genetically predisposed to an imbalance of enzymes within the cornea, making them more susceptible to oxidative damage by free radicals. This eventually distorts the shape of the cornea [3] and causes loss of vision.

Reported prevalence of KC in the general population varies (50-200 cases per 100,000 population) perhaps due to different diagnostic criteria [4]. It typically starts at the age of puberty, progresses in early years, and stabilizes during the fourth and fifth decades of life [4].

Depending on the severity of the disease, many treatment options like the use of eyeglasses and soft contact lenses have been devised. Laser in situ keratomileusis (LASIK) is contraindicated as a treatment because of the risk of progression to keratectasia [2, 5]. Artiflex iris-fixated anterior chamber phakic intraocular lens (ACIF-PIOL) implantation may, however, be considered a better alternative $[2,6]$. To our knowledge, one other paper has evaluated the use of ACIF-PIOLs in cases with progressive KC [7]. Therefore, we are presenting the case of a patient with progressive KC being successfully treated by ACIF-PIOL implantation.

\section{Case History}

A 24-year-old patient presented to the Hashmanis Hospital, Karachi, Pakistan on August 27, 2015 with a complaint of blurry vision which was progressively worsening. Refraction was performed using an autorefractometer (KR-800; Topcon Medical Systems, Inc., Fukuoka, Japan) and his results were: $-6 \mathrm{dpt},-3 \mathrm{dpt}$ at 60 degrees (right) and $-5 \mathrm{dpt},-2.5 \mathrm{dpt}$ at 100 degrees (left). His best corrected visual acuity was 6/12, bilaterally, which was measured using the Snellen's chart. Subsequently, an Oculus Pentacam (Pentacam HR, Oculus, Wetzlar, Germany) exam was performed. The $\mathrm{k} 1$ and $\mathrm{k} 2$ values of the right eye were found to be 47.7 and $49.2 \mathrm{dpt}$, whereas for the left eye, the values were 47.1 and $49.1 \mathrm{dpt}$, respectively. This exam was repeated 6 months later, which showed the patient had progressive KC bilaterally.

The doctor advised corneal collagen cross-linking (CXL) to stabilize his KC. He agreed to the terms and conditions and so according to the Dresden protocol [8], he got his CXL done on April 15, 2016. First, we anesthetized the eye with proparacaine drops. Secondly, we removed the epithelium by applying $18 \%$ alcohol for $18 \mathrm{~s}$, after which we gently brushed the epithelium off with a cannula. Thirdly, we soaked the stroma with riboflavin $0.1 \%$ every $1-$ $3 \mathrm{~min}$ for $30 \mathrm{~min}$. After this, we checked the patient's eye on a slit lamp to look for anterior chamber flare, which indicates the stroma taking up the riboflavin. Subsequently, we deliv- 
ered UVA light via the CCL-365 Vario system (Peschke Meditrade $\mathrm{GmbH}$ ) at $370 \mathrm{~nm}$ at 3.0 $\mathrm{mW} / \mathrm{cm}^{2}$ for $30 \mathrm{~min}$. This was given through a $9.0-\mathrm{mm}$ aperture that was $50 \mathrm{~mm}$ from the corneal apex.

This treatment halted the progression of the disease but did not correct his refractive error, due to which he was advised to wear glasses.

On July, 2016, he showed an interest in going for LASIK to get rid of his spectacles. Since LASIK was contraindicated in this patient, he was given the option of ACIF-PIOL implantation. The patient was counselled on the experimental nature of this procedure and was informed of the associated risks. The patient agreed to these conditions.

For his right eye, he was operated on August 11, 2016 with a -8.5 dpt ACIF-PIOL. His left eye was operated on August 16, 2016 with a -7.0 dpt ACIF-PIOL. The lens power was determined by the IOL Master (Carl Zeiss Meditec AG, Germany).

This patient achieved an uncorrected visual acuity of $6 / 12$ with a residual cylinder of -3 in both eyes. The postoperative $\mathrm{k} 1$ values of the right and left eye were 48 and $45.6 \mathrm{dpt}$, while the $\mathrm{k} 2$ values were found to be 49.5 and $48.0 \mathrm{dpt}$, respectively. The patient was satisfied and happy. His visual performance over an 11-month period is shown in Table 1. His Oculus Pentacam exam at 11 months post ACIF-PIOL implantation is shown in Figure 1. Figure 2 shows his anterior segment imaging at 1 month post implantation via an optical coherence tomography angiography (Angiovue, Optovue, Inc., Freemont, CA, USA) machine. Figure 3 shows his implanted ACIF-PIOL.

\section{Discussion}

Refractive surgery was contraindicated in those with KC in the past [9]. LASIK, one of the most successful refractive surgeries in the world, cannot be performed in such patients due to fear of keratectasia $[10,11]$. However, novel techniques have enabled the physician to cater to this important demographic as well. Lenses such as ACIF-PIOLs [12] and toric intraocular lenses [13] have been reported to be safe and effective in those with stable KC. However, a dearth of information is available in those with progressive KC. One report has shown these lenses to be safe and effective in those with progressive KC post CXL [7]. This report highlights a similar result and therefore, we recommend ACIF-PIOL implantation post CXL in those with progressive KC.

When implanting an anterior chamber PIOL, damaging the anterior chamber structures can be a worrisome complication. The corneal endothelium is especially prone to damage during such procedures. However, newer iris-fixated and angle-supported PIOLs cause minimal damage to the endothelium and therefore are well tolerated [14]. Furthermore, the ACIF-PIOL has no contact with the periphery of the anterior chamber due to a lack of angle support; this minimizes contact of the lens with the corneal endothelium. Lastly, danger of cataracts is also diminished as these ACIF-PIOLs are placed far from the lens [14]. These complications were not found in our case nor did we find any other complications like high intraocular pressure, halos, glare, or inflammation.

A normal cornea maintains its natural shape by cross-linkages amongst its constituent collagen fibers that keep it strong. The cornea has a natural tendency to create new collagen cross-links in response to the damage caused by smoking, diabetes mellitus, and aging [1]. 
Since the main ophthalmologic change occurring in the eye of patients affected by KC is corneal thinning and weakening, the case under study required an operative CXL of the cornea to be done as a first-line treatment. The greatest aim of CXL is to stop KC in its early phase and avoid its progression to a stage where corneal transplantation is the only treatment option left, which is not without its own adverse effects.

The number of clinical reports suggesting a stabilizing effect of CXL with significant yet variable improvement in corneal shape and visual function are rapidly increasing [15]. This noninvasive treatment involves saturating the cornea with ophthalmic riboflavin (vitamin $B_{2}$ ) solution, which is a photosensitizer, followed by UVA irradiation. New collagen crosslinks are induced by the photo-oxidative damage to stiffen and stabilize the cornea, which retards the progression of the disease [15]. The accompanying disorders like myopia and astigmatism, however, continue to exist, though not as severe as before. Use of spectacles and contact lenses is then recommended as a remedy to these. As these were unacceptable to the patient, an ACIF-PIOL was successfully offered as an alternative.

\section{Conclusions}

ACIF-PIOLs are indicated in those with stable KC. However, few studies have analyzed the effects of ACIF-PIOL post CXL in those with progressive KC. This report highlights the success of ACIF-PIOL post CXL in this patient.

\section{Statement of Ethics}

The study was approved by the Ethics Committee of Hashmanis Hospital.

\section{Disclosure Statement}

This manuscript is not under consideration anywhere else.

\section{References}

1 Pron G, Ieraci L, Kaulback K, et al: Collagen cross-linking using riboflavin and ultraviolet-A for corneal thinning disorders: an evidence-based analysis. Ont Health Technol Asses Ser 2011;11:1-89.

-2 Kato N, Toda I, Hori-Komai Y, et al: Phakic intraocular lens for keratoconus. Ophthalmology 2011;118:605-605.e602.

3 Gondhowiardjo T, van Haeringen N, Volker-Dieben H, et al: Analysis of corneal aldehyde dehydrogenase patterns in pathologic corneas. Cornea 1993;12:146-154.

4 Greenwald M, Scruggs B, Vislisel J, et al: Corneal Imaging: An Introduction. eyerounds.org, 2016, cited June 30, 2017.

5 Hashmani S, Hashmani N, Kumar S, et al: Reasons for refusing laser-assisted in situ keratomileusis in a Pakistani population. Cureus 2017;9.

6 Pineda R, Chauhan T: Phakic intraocular lenses and their special indications. J Ophthalmic Vis Res 2016;11:422-428. 
7 Izquierdo L, Henriquez M, McCarthy M: Artiflex phakic intraocular lens implantation after corneal collagen cross-linking in keratoconic eyes. J Refract Surg 2011;27:482-487.

8 Choi M, Kim J, Kim E, et al: Comparison of the conventional Dresden protocol and accelerated protocol with higher ultraviolet intensity in corneal collagen cross-linking for keratoconus. Cornea 2017;36:523-529.

-9 Vazirani J, Basu S: Keratoconus: current perspectives. Clin Ophthalmol 2013;7:2019-2030.

10 Hashmani S, Hashmani N, Rajani H, et al: Comparison of visual acuity, refractive outcomes, and satisfaction between LASIK performed with a microkeratome and a femto laser. Clin Ophthalmol 2017;11:1009-1014.

11 Hashmani N, Hashmani S, Hanfi A: Effect of age, sex, and refractive errors on central corneal thickness measured by Oculus Pentacam ${ }^{\circledR}$. Clin Ophthalmol 2017;11:1233-1238.

12 Guell J, Morral M, Malecaze F, et al: Collagen crosslinking and toric iris-claw phakic intraocular lens for myopic astigmatism in progressive mild to moderate keratoconus. J Cataract Refract Surg 2012;38:475-484.

13 Fadlallah A, Dirani A, El Rami H, et al: Safety and visual outcome of Visian toric ICL implantation after corneal collagen cross-linking in keratoconus. J Refract Surg 2013;29:84-89.

14 Munos G, Belda L, Albarran-Diego C, et al: Artiflex toric phakic intraocular lens implantation in congenital nystagmus. Case Rep Ophthalmol 2011;2:273-278.

$>15$ Wollensak G, Spoerl E, Seiler T: Riboflavin/ultraviolet-a-induced collagen crosslinking for the treatment of keratoconus. Am J Ophthalmol 2003;135:620-627. 


\section{Case Reports in Ophthalmology}

Case Rep Ophthalmol 2017;8:482-488
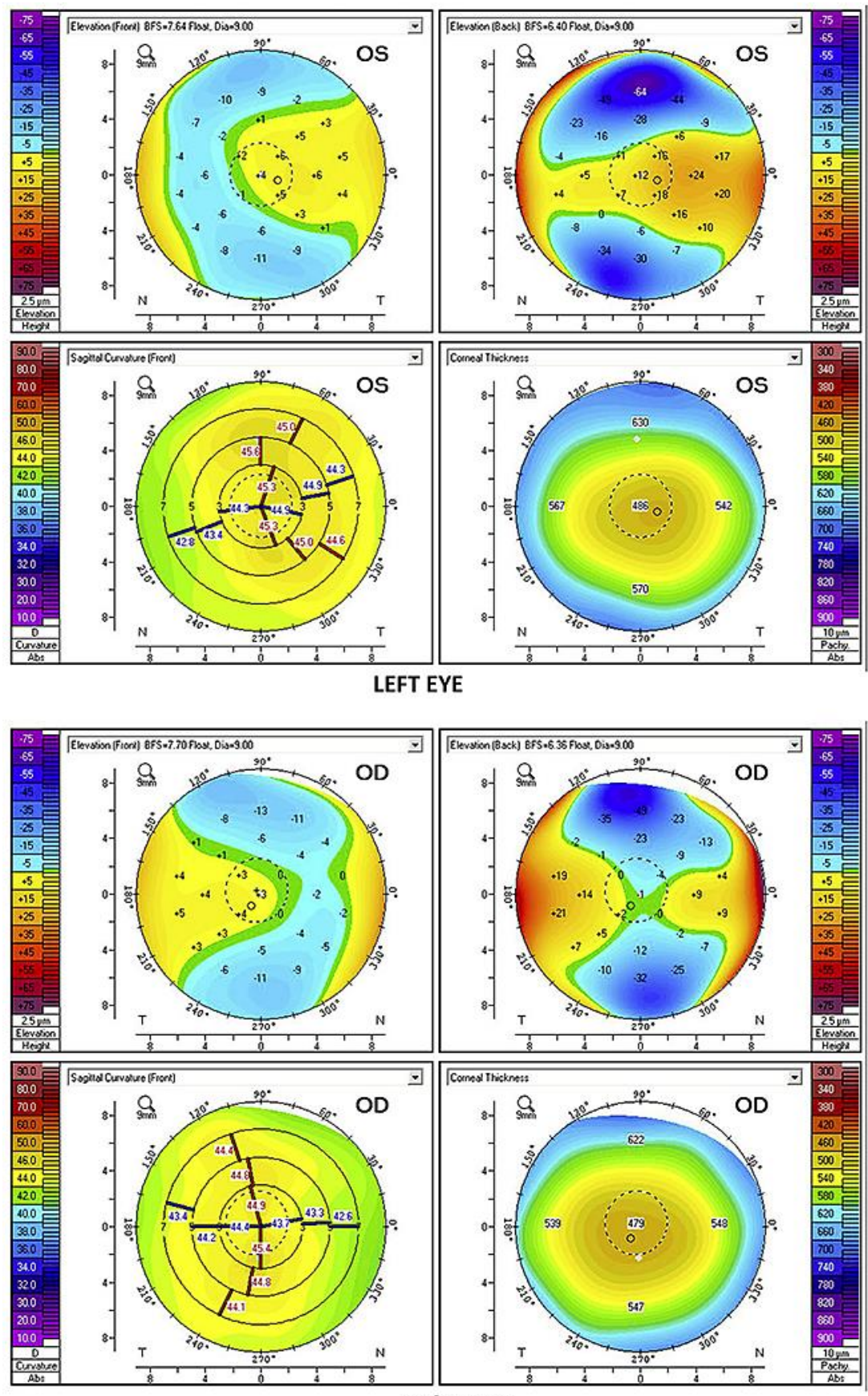

RIGHT EYE

Fig. 1. Oculus Pentacam examination at month 11 after ACIF-PIOL implantation. 


\section{Case Reports in Ophthalmology}

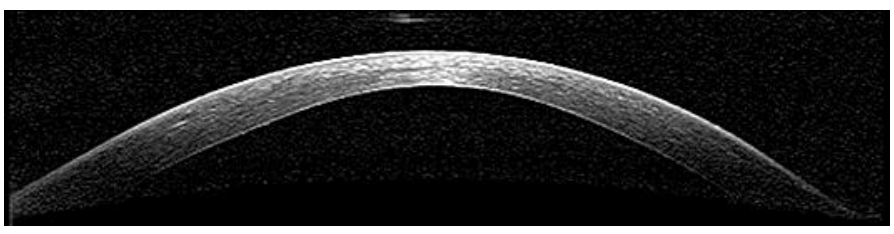

Fig. 2. Anterior segment imaging at month 1 post ACIF-PIOL implantation.

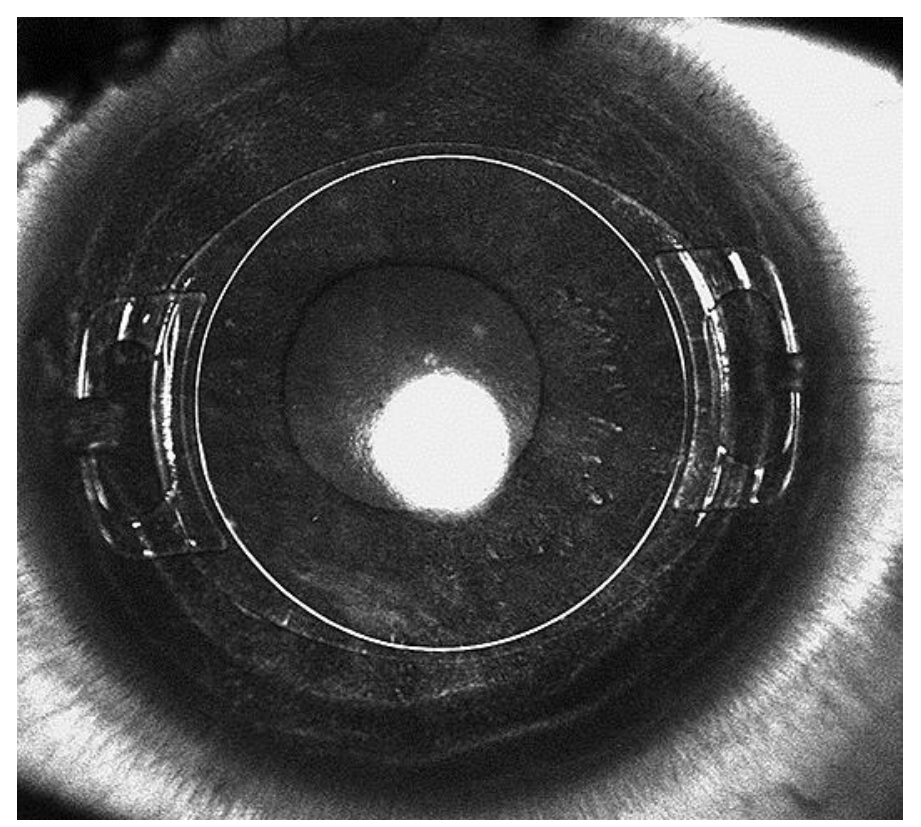

Fig. 3. The implanted ACIF-PIOL.

Table 1. Visual performance over time

\begin{tabular}{|c|c|c|c|c|c|c|c|c|c|c|}
\hline \multirow[b]{3}{*}{ Parameters } & \multirow{2}{*}{\multicolumn{2}{|c|}{ Preoperative }} & \multicolumn{8}{|c|}{ Postoperative } \\
\hline & & & \multicolumn{2}{|l|}{1 day } & \multicolumn{2}{|l|}{1 week } & \multicolumn{2}{|l|}{6 months } & \multicolumn{2}{|c|}{11 months } \\
\hline & left & right & left & right & left & right & left & right & left & right \\
\hline Visual acuity & $6 / 12^{*}$ & $6 / 12^{*}$ & $6 / 12^{* *}$ & $6 / 12^{* *}$ & $6 / 12 * *$ & $6 / 12^{* *}$ & $6 / 12 * *$ & $6 / 12^{* *}$ & $6 / 12^{* *}$ & $6 / 12 * *$ \\
\hline Sphere, dpt & -5.00 & -6.00 & 0.25 & 0.25 & 0.25 & 0.25 & 0.25 & 0.25 & 0.00 & 0.50 \\
\hline Cylinder, dpt & -2.50 & -3.25 & -3.00 & -3.25 & -3.00 & -3.25 & -3.00 & -3.25 & -2.00 & -3.00 \\
\hline Spherical equivalent, $\mathrm{dpt}$ & -6.25 & -7.63 & -1.25 & -1.38 & -1.25 & -1.38 & -1.25 & -1.38 & -1.00 & -1.00 \\
\hline
\end{tabular}

* Best corrected visual acuity; ${ }^{* *}$ uncorrected visual acuity. 